\title{
A Vision to Face Covid-19 pandemic and Future Risks Through Artificial Intelligence
}

\author{
Malek Khalaf Albzeirat ${ }^{1,2 *}$, Nik Noriman Zulkepli ${ }^{1,2}$, Haitham Qaralleh $^{3}$ \\ ${ }^{1}$ Center of Excellence Geopolymer and Green Technology (CEGeoGTech), Universiti Malaysia Perlis (UniMAP), Kompleks Pengajian \\ Jejawi 2, 02600 Arau, Perlis, Malaysia; ${ }^{2}$ Center of Excellence for Sport and engineering Research, University Malaysia Perlis, Kampus \\ Alam Pauh Putra, 02600 Arau, Perlis Malaysia; ${ }^{3}$ Department of Medical Laboratory Sciences, Mutah University, Mutah, Karak, Jordan \\ *Corresponding Author: eng.malekkhalaf@gmail.com
}

Received: 29-03-2020

Revised: 6-4-2020

Published: $10-4-2020$

Keywords:

Covid-19, Pandemic,

Future Risks,

Cognitive Fission Theory,

Artificial Intelligence

\begin{abstract}
Coved-19 pandemic is spreading fear among the world in several aspects such as health, economic, international relations, political stability, and social stability. It emerged suddenly and attacked the world in a short period without warning. Details about the virus such as the source, symptoms, transmission, diagnosis and treatment are still incomplete. Subsequently, more than one million people have died and huge economic losses. In order to avoid this issue in future, this paper aims to focus on artificial intelligence in predicting and tracking viral pandemic Disease and to control similar future risks using artificial intelligence, algorithms and cognitive fission theory.
\end{abstract}

Cite this article as: Albzeirat, M.K., Zulkepli, N.N., Qaralleh, H. (2020) A Vision to Face Covid-19 pandemic and Future Risks Through Artificial Intelligence. Journal of basic and applied Research in Biomedicine, 6(1): 15-20 This work is licensed under a Creative Commons Attribution 4.0 License. You are free to copy, distribute and perform the work. You must attribute This work is licensed under a Creative Commons Attribution 4.1
the work in the manner specified by the author or licensor.

\section{INTRODUCTION}

Coronaviruses are a large family of viruses that cause illness ranging from common cold to life threatening disease. Historically, the world has known specific types of coronaviruses. In 1960, strain B814 was isolated as the first human coronavirus that causes infection in humans. In 1966, virus 229E (HCoV-229E) has been isolated and Strain OC43 has also been isolated in the same year. These viruses, despite their seriousness, did not constitute the stage of spreading within the description of the epidemic, until the beginning of the new millennium. In general, these coronaviruses had limited effects and did not cause a global pandemic. But, in 2003 a new type of these viruses appeared. On April 12, 2003, scientists working at the Michael Smith Genome Sciences Centre in Vancouver have come up with a complete genetic map of a new Coronavirus strain (Marra et al., 2003). Phylogenetic analysis of this virus indicated a high probability that this strain is linked to SARS and it is originated in bats and spread to humans either directly or through animals held in Chinese markets (Rihtarič et al., 2010). Severe acute respiratory syndrome SARS Coronavirus emerged in early 2003 to cause a very severe acute respiratory syndrome, which eventually resulted in a $10 \%$ case-fatality rate (Chiu et al., 2005, Bastien et al., 2005).

Owing to excellent public health measures that isolated focused cases and their contacts, and the use of supportive therapies, the epidemic was suppressed to the point that further cases have not appeared since 2005. However, it was the first epidemic caused by coronaviruses, it represented a major turning point in the effectiveness of these viruses and their danger to humans, as the description moved to epidemiological expression. Ten years later, studies indicated that the source of Middle East respiratory syndrome (MERS-CoV) is unknown Coronaviruses (Memish et al., 2013; Assiri et al., 2013, Haagmans et al., 2014), their source may be camels and bats, and these are indications that animals are a source of viruses that infect the respiratory system. It is noted that this infection was limited and did not rise to the level of pandemic. In the past five years there have been fears of spread of SARS or Middle East respiratory syndrome as a pandemic (Al-Tawfiq \& Memish, 2016).

Scientists had concerns about a pandemic caused by Coronaviruses, but they were not supported by scientific evidence. Fears were directed towards the emergence of these viruses from pigs, birds, bats, camels or cows (YusoF, 2017; Corman et al., 2018; Fan et al., 2019), but these fears were facing a silent and unpredictable enemy. In general, coronaviruses are enveloped by protein, positivesense, single-stranded RNA genome (26-32kb). Coronaviruses belongs to Nidovirales order and are classified in four genera (alpha, beta, gamma and delta). Human coronaviruses are members of alpha and beta genera. Coronaviruses are so called due to the crown like spikes projection on the outer surface of the virus (Zhong et al., 2003)

Through comparisons between the Covid 19 virus and other coronaviruses, there are basic points that 
provide a starting point for studying it in an accelerated manner, which contributes to controlling the pandemic or reducing the time to access medical drugs for future treatment. The virus is called SARS-CoV-2 by the International Committee on Taxonomy of Viruses (ICTV). It belongs to Beta coronavirus genus. According to the phylogenetic tree, SARS-CoV-2 is highly related to SARS-coronaviruses. About $80 \%$ of SARS-CoV-2 genome is identical to the SARS-like bat $\mathrm{CoV}$. Structural proteins are encoded by the four structural genes, including spike (S), envelope $(\mathrm{E})$, membrane $(\mathrm{M})$ and nucleocapsid $(\mathrm{N})$ genes. Also, the mechanism of the entry to the cells appears to be identical with the SARS-CoV since both of them use the same receptor known as angiotensin-converting enzyme 2 (Raj et al., 2013 and Wang et al., 2013)

Covid-19 appeared suddenly without warning. The fears that indicated the possibility of the emergence of newly and highly dangerous coronaviruses on humans have been realized. Precisely on December 2019, several cases have been diagnosed with unknown pneumonia in Wuhan, China. This unidentified pneumonia was highly contagious, and about 1.5 million and half confirmed cases has been recorded globally (9/4/2020) with fatality rate of $6 \%$. According to the molecular analysis, the causative agent was identified as a new generation of coronavirus named COVID-19. Then, the World Health Organization (WHO) declared Covid-19 as pandemic on March 2020. With this description from the WHO, Covid 19 becomes the first coronavirus that infects people, causing a global pandemic.

The rate of transmission of SARS-CoV-2 is higher than SARS-CoV and MERS-CoV. Recent reports showed that this pandemic is expanding, and the virus infect both human and animals. In an accelerating development, reports recorded the first infection of a cat in Belgium at the end of March, 2020 and a case of infected tiger was recorded in the United States of America at the beginning of April, 2020 (BBC News, 2020). The transition of covid-19 from epidemic to pandemic can be summarized by diagram 1 .

From Figure 1, it is possible to look deeper than the current reality of the pandemic of Covid 19. It is noted that short period was enough to transit the epidemic to pandemic (less than 8 years). Subsequently, emergence of new other types of coronavirus is predictable in a short period of time. This problem requires new scientific methods that can predict the emergence of any new viruses. Artificial intelligence can play a significant role to solve the current problem and any future problems.

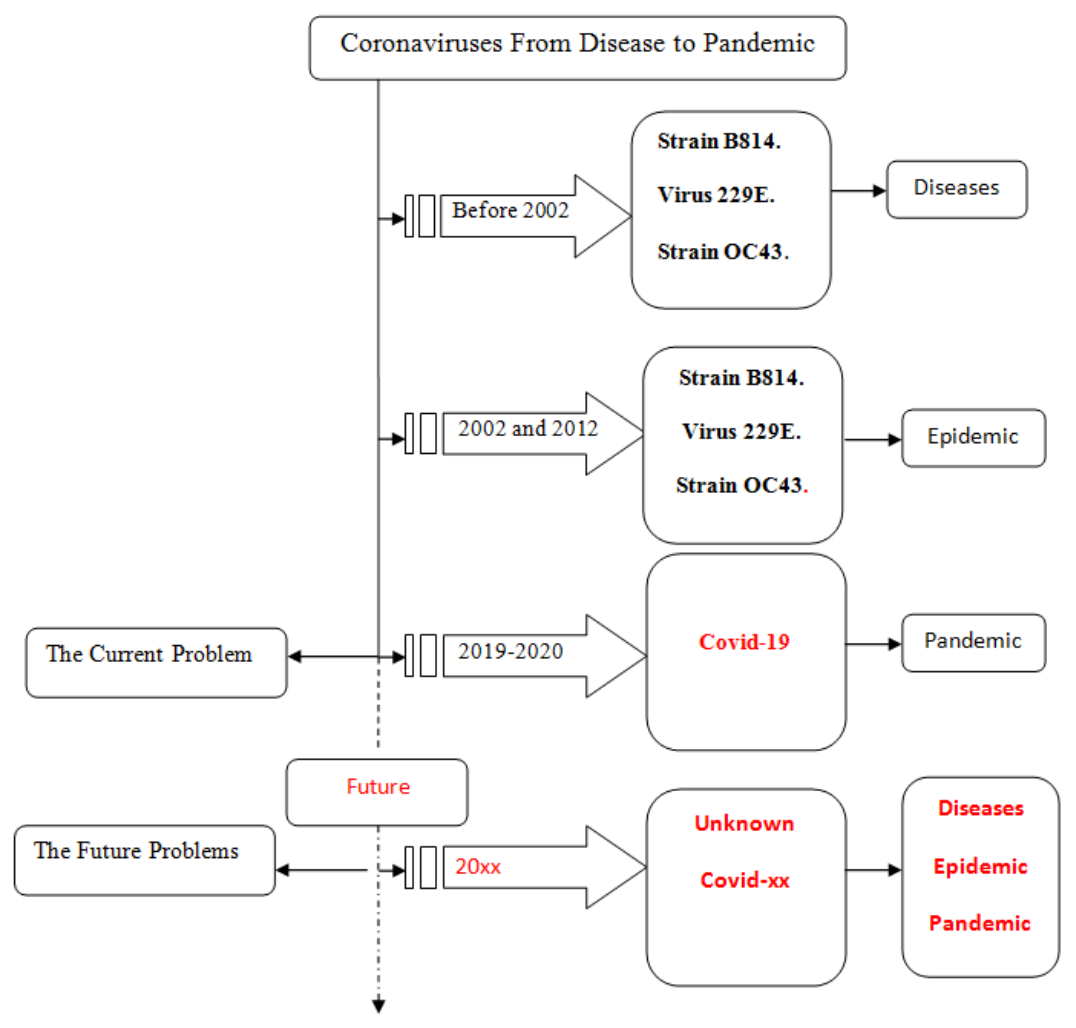

Diagram 1: Chronological Evolution of COVID-19 pandemic 


\section{COVID-19: HOSTS, TRANSMISSION, SYMPTOMS, DIAGNOSIS, TREATMENT AND PREVENTION}

However, the natural reservoir of this virus still unknown, scientist believes it is a zoonotic disease and it transmitted from animals such as rats, bats, pigs, or some other source. The similarity of Covid19 sequence with those viruses that originated from bats supports that this virus is originated from bats. Other reports suggested raccoon dog to be the susceptible host. In addition to the contact with infected animals, the rout of transmission of Covid19 appears to be limited to person-to-person transmission and direct contact with tools and surfaces contaminated with the nasal discharge and sputum of infected subject (Shereen et al., 2020).

The common symptoms caused by 2019-nCoV were fever, dry cough and sometime dyspnoea. Other Symptoms such as nausea, vomiting and diarrhea were also reported. On the other hand, the laboratory signs that have been reported were Leukopenia (63\%), Thrombocytopenia, increase in aspartate aminotransferase (AST) and alanine aminotransferase (ALT) levels. Increasing in creatine kinase or lactate dehydrogenase enzymes. Also, increasing in C-reactive protein (CRP) and erythrocyte sedimentation rate (ESR) (Lupia et al., 2020).

Covid-19 can be diagnosed by real-time RT-PCR, Chest $\mathrm{x}$-ray and computer tomography (CT). the acceptable respiratory specimens that can be used such as nasopharyngeal swab, sputum, lower respiratory tract aspirates (Shereen et al., 2020 and Lupia et al., 2020).

Up to date, there is no specific antiviral drugs for COVID-19. Various antiviral drugs such as lopinavir/ritonavir and ribavirin have shown significant inhibitory effect against the virus. The unapproved antiviral drug called Remdesivir found to decrease the viral load. convalescent plasma, interferon-beta/ribavirin combination therapy have been used to support the immune system (Shereen et al., 2020 and Lupia et al., 2020).

The prevention strategies such as avoiding traveling to infected area and avoiding eating wild animals. Those who were in infected area, they should perform 14 days self-surveillance. Those who are in contact with the confirmed cases, personal protective tools should be used and wasted properly. While there is no cure for this virus infection, it is wise to try to stop the spread of Covid-19 through isolation of infected area.

\section{COVID-19 AND ISOLATION PROCEDURE}

The behavioral measure in the face of COVID19 includes, with some variation among countries, conducting self-surveillance. Historically, this technique was applied previously as an effective technique to limit the spread of the pandemic. Islamic legislation during time of Khalifa Omar used this technique in middle east to avoid the infection with plaque. Through isolation, China has demonstrated the ability to manage the epidemic at its early stages of spread, but some countries, that have hesitated to implement the isolation protocol, have faced uncontrolled pandemic such as Italy and Iran.

Now many countries continued to take strict measures to do isolation, for example, Philippian, Jordan and Malaysia. Isolation is more effective whenever it is strict with minimum exceptions, and the situation has arrived in Jordan to announce a curfew. Isolation requires a capacity to control society and an ability to cope with the consequences of this total closure in the future.

Understanding that the ability of the virus to remain effective in the external environment or in the hosts should be take into consideration. For instance, isolation should be temperature dependent since most viruses are sensitive to temperature higher than 25C. Strict performance of isolation should be more than the incubation period of this virus in which this period was found to be more than 14 days and up to 26 days. Also, the isolation should consider the expected period of maximum spreading that might be appeared between April and May. Moreover, isolation should be applied strictly on people who are at risk of infections such as the most elderly, children and those with chronic respiratory diseases. Most important, isolation procedures should be extended until the number of confirmed cases reach zero for up to 14 days.

However, the isolation procedure is very effective protocol, finding specific antiviral drugs and vaccine should be made as soon as possible. Most important is to find new diagnostic methods and tools faster than the currently used methods.

Infection rate, global health reports and scientists predicted that the rate of infection of COVID-19 might reach $40 \%$ of the world's population, although there is no evidence for this percentage, but it means that there is a probability of more than 2.5 billion people of the world will be infected with COVID-19. Reports also indicated that the death rate may increase, and this means that the number of deaths may reach up to about 50 million people. These predictable numbers are closed to the number of people killed in World War II, which reinforces the description of the pandemic as 'world war'. 
Several negative effects are associated with the isolation procedure. Isolation causes an almost complete halt to the global trade. It could seriously harm all countries regardless their economic situation. Local isolation could be harm to the local economy if the epidemic spreads are out of control. The epidemic may deepen poverty and hunger in the world especially in unstable regions such as yamen, syria, libea, Gazza, Afghanistan and others. Isolation has been reported to increase cases of psychological behavior and suicide (Joiner et al., 2005).

\section{ARTIFICIAL INTELLIGENCE (AI) AND FUTURE VISION}

The world is expected to be able to control Covid19 pandemic through isolation, but this does not mean that it is the best option. Trials in designing and developing smart devices that rely on the Internet of things and smart applications in managing epidemics is started. Using the Internet of Things (IoT) to monitor the spread of the disease and then facilitate immediate reporting and alarming system. The use of artificial intelligence (AI) in the process of predicting the evolution of any viruses through genetic analysis and analysis of interactions between viruses with each other or with the surrounding environment. Application of fuzzy models in studying the behaviour of viruses and to anticipate what might happen in future (Saha et al., 2017).

For effective AI, comprehensive collection and integration system should be performed. Data can be collected from scientific literature, social media, newspaper, government declarations and other sources. Integration of these data could help also in raising the health education among people who are under the risk of infections.

AI can be applied here also to facilitate the detection of new viral infections. The integration of all known data about the previously known viruses such as symptoms and target tissues could facilitate the finding of any emerged viruses with unusual symptom. Faster diagnosis can be mad using AI as a model. Integration of the X-rays image of patients along with the specific symptoms could make the diagnosis faster. The integration of data about the incubation period, the rout of transmission, the ports of entry, traveling and culture practices could be critical to control the spreading of the diseases in term of when and how (Ahuja et al., 2019).
$\mathrm{AI}$ and data integration about the previously known virus can facilitate the treatment and the finding of new therapy. Also, integration of data about the currently used antivirals and immunomodulators drugs such as mechanism of action, physiological and side effects, chemical structure, doses and precautions could be critical for accelerating the treatment. Prediction of a geographical area where new infected patients can emerge could be controlled by AI. The integration of data about the sensitivity of COVID-19 virus and previously similar known viruses to the environmental conditions, reservoirs and hosts, social and religious practices could help to control the spreading of the pandemic.

\section{RECOMMENDATION AND CONCLUSION}

In the Covid 19 pandemic, attempts and uses of smart technologies to manage the pandemic has been started. The Chinese and Japanese used robots for surveillance. Countries like Qatar, Indonesia, and Jordan also use drones to monitor the isolation process. New applications related to remote temperature checks and thermal monitoring cameras have also emerged to identify injuries in public places. In addition to that, locator and tracker applications through smart phones have been used. Smart robots can be used in hospitals, mobile clinics and smart medical rooms.

Although many researches in AI have been started, the most attention must be given to smart simulation that focus on future prediction. This perception relates to the future of virus prediction and how to deal with it. This vision can be started through genetic and neuronal algorithms and deep learning. A simulation that can answer many questions. In this study, a suggested diagram (2) can be considered as basic vision to solve this problem.

Despite the positive role that artificial intelligence plays in the future to deal with viruses and pandemics, it includes a real risk. The integration of this Knowledge Sequence is like nuclear fission, it can be said that any knowledge structure can be described as (Theory of Cognitive Fission). Therefore, the risks and challenges facing this future vision in combating viruses and epidemics should be considered in order to prevent using this knowledge to build a biological weapon. 


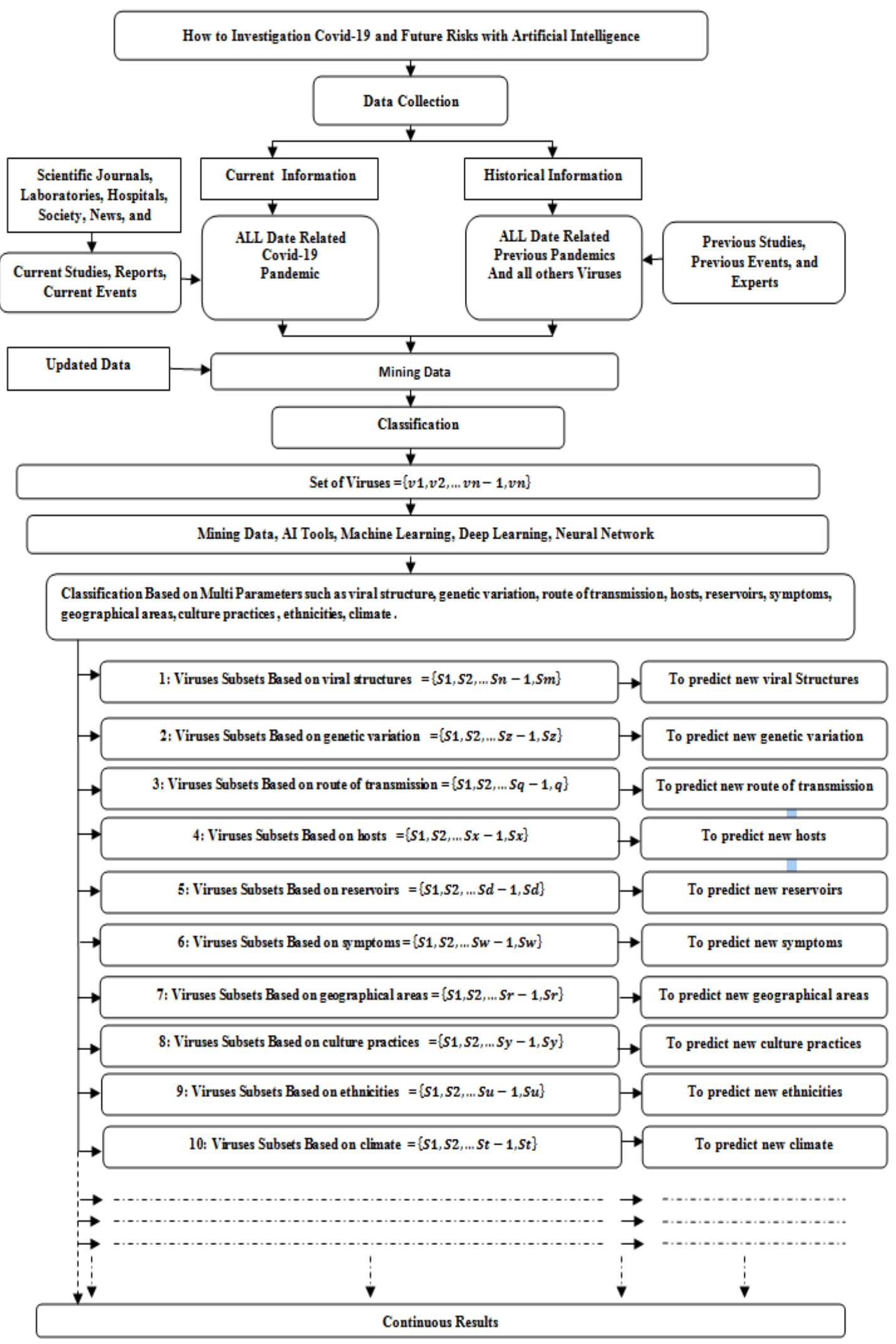

Diagram 2: suggested application of AI to overcome COVID-19 pandemic. 


\section{REFERENCES}

Ahuja, A. S. (2019). The impact of artificial intelligence in medicine on the future role of the physician. PeerJ, 7, e7702.

Al-Tawfiq, J. A., \& Memish, Z. A. (2016). The middle east respiratory syndrome coronavirus respiratory infection: An emerging infection from the Arabian Peninsula. In The Microbiology of Respiratory System Infections (pp. 55-63). Academic Press.

Assiri, A., McGeer, A., Perl, T. M., Price, C. S., Al Rabeeah, A. A., Cummings, D. A., ... \& Madani, H. (2013). Hospital outbreak of Middle East respiratory syndrome coronavirus. New England Journal of Medicine, 369(5), 407-416.

Bastien, N., Anderson, K., Hart, L., Caeseele, P. V., Brandt, K., Milley, D., \& Li, Y. (2005). Human coronavirus NL63 infection in Canada. The Journal of infectious diseases, 191(4), 503-506.

BBC News, (2020) Coronavirus: Tiger at Bronx Zoo tests positive for Covid-19. Retrieved at 4/9/2020 Available at https://www.bbc.com/news/world-uscanada-52177586

Chiu, S. S., Hung Chan, K., Wing Chu, K., Kwan, S. W., Guan, Y., Man Poon, L. L., \& Peiris, J. S. M. (2005). Human coronavirus NL63 infection and other coronavirus infections in children hospitalized with acute respiratory disease in Hong Kong, China. Clinical infectious diseases, 40(12), 1721-1729.

Corman, V. M., Muth, D., Niemeyer, D., \& Drosten, C. (2018). Hosts and sources of endemic human coronaviruses. In Advances in virus research (Vol. 100, pp. 163-188). Academic Press.

Fan, Y., Zhao, K., Shi, Z. L., \& Zhou, P. (2019). Bat Coronaviruses in China. Viruses, 11(3), 210.

Haagmans, B. L., Al Dhahiry, S. H., Reusken, C. B., Raj, V. S., Galiano, M., Myers, R., ... \& Ghobashy, H. (2014). Middle East respiratory syndrome coronavirus in dromedary camels: an outbreak investigation. The Lancet infectious diseases, 14(2), 140-145.

Joiner Jr, T. E., Brown, J. S., \& Wingate, L. R. (2005). The psychology and neurobiology of suicidal behavior. Annu. Rev. Psychol., 56, 287-314.

Lupia T., S. Scabini, S. Mornese Pinna, G. Di Perri, FG De Rosa, and S. Corcione, 2019 novel coronavirus (2019-nCoV) outbreak: A new challenge (2020),"Journal of Global Antimicrobial Resistance (21): 22-27

Marra, M. A., Jones, S. J., Astell, C. R., Holt, R. A., Brooks-Wilson, A., Butterfield, Y. S., ... $\&$ Cloutier, A. (2003). The genome sequence of the SARS-associated coronavirus. Science, 300(5624), 1399-1404.

Memish, Z. A., Mishra, N., Olival, K. J., Fagbo, S. F., Kapoor, V., Epstein, J. H., ... \& Kapoor, A. (2013). Middle East respiratory syndrome coronavirus in bats, Saudi Arabia. Emerging infectious diseases, 19(11), 1819.

Raj VS, Mou H, Smits SL, Dekkers DH, Müller MA, Dijkman R, et al. Dipeptidyl peptidase 4 is a functional receptor for the emerging human coronavirus-EMC. Nature 2013;495(7440):251-4

Rihtarič, D., Hostnik, P., Steyer, A., Grom, J., \& Toplak, I. (2010). Identification of SARSlike coronaviruses in horseshoe bats (Rhinolophus hipposideros) in Slovenia. Archives of virology, 155(4), 507514.

Saha, H. N., Auddy, S., Pal, S., Kumar, S., Pandey, S., Singh, R., ... \& Saha, S. (2017, August). Health monitoring using internet of things (iot). In 2017 8th Annual Industrial Automation and Electromechanical Engineering Conference (IEMECON) (pp. 69-73). IEEE.

Shereen, M. A., Khan, S., Kazmi, A., Bashir, N., \& Siddique, R. (2020). COVID-19 infection: origin, transmission, and characteristics of human coronaviruses. Journal of Advanced Research, 24: 91-98

Wang N, Shi X, Jiang L, Zhang S, Wang D, Tong $P$, et al. Structure of MERS-CoV spike receptor-binding domain complexed with human receptor DPP4. Cell Res 2013;23(8):986.

Yusof, M. F., Queen, K., Eltahir, Y. M., Paden, C. R., Al Hammadi, Z. M. A. H., Tao, Y., ... \& Mohamed, M. S. A. E. (2017). Diversity of Middle East respiratory syndrome coronaviruses in 109 dromedary camels based on full-genome sequencing, $\mathrm{Abu}$ Dhabi, United Arab Emirates. Emerging microbes \& infections, 6(1), 1-10.

Zhong N, Zheng B, Li Y, Poon L, Xie Z, Chan K, et al. Epidemiology and cause of severe acute respiratory syndrome (SARS) in Guangdong, People's Republic of China, in February, 2003. The Lancet 2003;362(9393):1353-8. 